\title{
Constitutive Expression of OsIAA9 Affects Starch Granules Accumulation and Root Gravitropic Response in Arabidopsis
}

\author{
Sha Luo, Qianqian Li, Shanda Liu, Nicholaas M. Pinas, Hainan Tian and Shucai Wang* \\ Key Laboratory of Molecular Epigenetics of MOE, Northeast Normal University, Changchun, China
}

\section{OPEN ACCESS}

Edited by: Frantisek Baluska, University of Bonn, Germany

Reviewed by: Xuelin Wu,

Harvey Mudd College, USA Jitendra Paul Khurana, University of Delhi South Campus,

India

*Correspondence: Shucai Wang wangsc550@nenu.edu.cn

Specialty section: This article was submitted to Plant Evolution and Development, a section of the journal

Frontiers in Plant Science

Received: 08 September 2015 Accepted: 04 December 2015 Published: 22 December 2015

Citation:

Luo S, Li Q, Liu S, Pinas NM, Tian H and Wang S (2015) Constitutive Expression of OsIAA9 Affects Starch Granules Accumulation and Root Gravitropic Response in Arabidopsis. Front. Plant Sci. 6:1156. doi: 10.3389/fp/s.2015.01156
Auxin/Indole-3-Acetic Acid (Aux/IAA) genes are early auxin response genes ecoding short-lived transcriptional repressors, which regulate auxin signaling in plants by interplay with Auxin Response Factors (ARFs). Most of the Aux/IAA proteins contain four different domains, namely Domain I, Domain II, Domain III, and Domain IV. So far all Aux/IAA mutants with auxin-related phenotypes identified in both Arabidopsis and rice (Oryza sativa) are dominant gain-of-function mutants with mutations in Domain II of the corresponding Aux/IAA proteins, suggest that Aux/IAA proteins in both Arabidopsis and rice are largely functional redundantly, and they may have conserved functions. We report here the functional characterization of a rice Aux/IAA gene, OsIAA9. RTPCR results showed that expression of OsIAA9 was induced by exogenously applied auxin, suggesting that OsIAA9 is an auxin response gene. Bioinformatic analysis showed that OsIAA9 has a repressor motif in Domain I, a degron in Domain II, and the conserved amino acid signatures for protein-protein interactions in Domain III and Domain IV. By generating transgenic plants expressing GFP-OsIAA9 and examining florescence in the transgenic plants, we found that OsIAA9 is localized in the nucleus. When transfected into protoplasts isolated from rosette leaves of Arabidopsis, OsIAA9 repressed reporter gene expression, and the repression was partially released by exogenously IAA. These results suggest that OsIAA9 is a canonical Aux/IAA protein. Protoplast transfection assays showed that OsIAA9 interacted ARF5, but not ARF6, 7, 8 and 19. Transgenic Arabidopsis plants expressing OsIAA9 have increased number of lateral roots, and reduced gravitropic response. Further analysis showed that OsIAA9 transgenic Arabidopsis plants accumulated fewer granules in their root tips and the distribution of granules was also affected. Taken together, our study showed that OsIAA9 is a transcriptional repressor, and it regulates gravitropic response when expressed in Arabidopsis by regulating granules accumulation and distribution in root tips.

Keywords: OsIAA9, auxin signaling, gravitropism, lateral root formation, Arabidopsis thaliana, Oryza sativa 


\section{INTRODUCTION}

In Arabidopsis, Aux/IAA genes are one of the early auxin response gene families (Hagen and Guilfoyle, 2002). Aux/IAA proteins are short-lived transcription repressors that involve in the regulation of auxin signaling (Guilfoyle and Hagen, 2007; Guilfoyle, 2015). Most of the Aux/IAA proteins contain four conserved domains, namely, Domain I, Domain II, Domain III, and Domain IV. Domain I is an active repression domain containing a conserved LxLxL motif. Domain II contains a conserved degron and is responsible for the stability of Aux/IAA proteins. Domains III and IV are similar to the conserved $\mathrm{C}$-terminal dimerization domain of Auxin Response Factors (ARFs), and are required for homo dimerization among Aux/IAA proteins, and hetero dimerization between Aux/IAA proteins and ARFs (Ulmasov et al., 1997, 1999; Ramos et al., 2001; Tiwari et al., 2001, 2003, 2004; Dreher et al., 2006; Nanao et al., 2014).

Aux/IAA proteins regulate auxin signaling by interplay with ARFs (Guilfoyle and Hagen, 2007; Guilfoyle, 2015). When cellular auxin levels are low, Aux/IAA proteins are stable, and they can form dimers with ARF activators that bound on the TGTCTC Auxin Response Elements (AuxREs) in the promoter regions of the auxin response genes, thus inhibiting the expression of auxin response genes. Elevated cellular auxin levels will result in the activation of auxin receptor TIR1 (Dharmasiri et al., 2005; Kepinski and Leyser, 2005), leading to the ubiquitylation and then degradation of Aux/IAA proteins via the $26 \mathrm{~S}$ proteasome (Tan et al., 2007), thus allowing the ARF activators to activate auxin response genes (Ulmasov et al., 1999; Tiwari et al., 2001, 2004; Guilfoyle and Hagen, 2007).

So far all the aux/iaa mutants identified in Arabidopsis, including single T-DNA insertion mutants, double, and triple mutants of closely related Aux/IAA genes, showed no visible developmental defects (Overvoorde et al., 2005), suggesting that $A u x / I A A$ genes are largely redundant functionally. On the other hand, several different types of phenotypes were observed in the dominant aux/iaa mutants. for example, the iaa18-1 mutant has aberrant cotyledon placement in embryos (Ploense et al., 2009), the axr2-1/iaa7 mutant has agravitropic root and shoot growth, and a short hypocotyl and stem (Nagpal et al., 2000), the iaa16-1 mutant has restricted adult plant growth and abolished fertility in homozygous (Rinaldi et al., 2012), the iaa 18 and iaa 28 mutants are severely defective in lateral root formation (Rogg et al., 2001; Uehara et al., 2008), and the slr-1/iaa14 mutant completely lacks lateral roots (Fukaki et al., 2002). Some of the dominant gain-of-function iaa mutants even have opposite phenotypes. For example, the axr3/iaa17 mutant has defects in root hair development, while shy2/iaa3 mutant has longer root hairs (Knox et al., 2003). The shy2/iaa3 mutant also has other phenotypes including enlarged cotyledons, short hypocotyls, and altered auxin-regulated root development (Tian and Reed, 1999; Tian et al., 2002). These results suggest that stability of Aux/IAA proteins is crucial for their functions in regulating plant growth and development.

Consistent with the fact that Aux/IAA protein are unstable proteins, Arabidopsis transgenic plants expressing wild type Aux/IAA genes from Arabidopsis and grape (Vitis vinifera) are also morphological indistinguishable from wild type plants (Park et al., 2002; Fujita et al., 2012; Kohno et al., 2012). However, auxin-related phenotypes were observed in Arabidopsis transgenic plants expressing mutated $A u x / I A A$ gene with a mutation in Domain II, or $A u x / I A A$ gene lacking Domain II (Park et al., 2002; Fukaki et al., 2005; Sato and Yamamoto, 2008).

Phenotypic changes were observed in knock-down mutants of Aux/IAA genes in tomato (Solanum lycopersicum) (Wang et al., 2005a; Bassa et al., 2012; Deng et al., 2012; Su et al., 2014), and expressing wild type PtrIAA14.1, a poplar Aux/IAA gene in Arabidopsis resulted in phenotypic changes (Liu et al., 2015), suggesting the functions of $A u x / I A A$ from at least some plant species may different from that in Arabidopsis.

In rice (Oryza sativa), however, all the identified Aux/IAA mutants with auxin-related phenotypes are dominant gainof-function mutants with mutations in the Domain II of corresponding Aux/IAA proteins (Jun et al., 2011; Zhu et al., 2011; Kitomi et al., 2012), and auxin-related phenotypes were also observed in transgenic rice plants expressing mutated or dominant mutation-type rice $A u x / I A A$ genes (Nakamura et al., 2006; Song and $\mathrm{Xu}, 2013$ ), suggesting that Aux/IAA proteins in rice may regulate auxin signaling in a way similar to those in Arabidopsis.

We report here the characterization of OsIAA9, a rice Aux/IAA gene. Expression of OsIAA9 was greatly induced by exogenously supplied IAA. Bioinformatics and protoplast transfection assay results showed that OsIAA9 is a canonical Aux/IAA protein. When expressed in Arabidopsis in a wild type form, however, OsIAA9 affected lateral root formation and root gravitropic response.

\section{MATERIALS AND METHODS}

\section{Plant Materials and Growth Conditions}

The Japonica rice (Oryza sativa) variety Nipponbare was used for Auxin treatment and OsIAA9 gene cloning. The Arabidopsis thaliana (Arabidopsis) ecotype Columbia-0 (Col-0) was used for plant transformation and protoplasts isolation. DR5:GUS transgenic plants were in Col background (Wang et al., 2005b).

For auxin treatment and RNA isolation from rice seedlings, rice seeds were germinated and grown on water for $10 \mathrm{~d}$, and treated with $10 \mu \mathrm{M}$ IAA for $4 \mathrm{~h}$ before RNA was isolated. For plant transformation and protoplasts isolation, Arabidopsis seeds were sown directly into soil pots and kept in a growth room. For phenotypic analysis, Arabidopsis seeds were sterilized and sown on $0.8 \%(\mathrm{w} / \mathrm{v})$ phytoagar solidified $1 / 2$ MS (Murashige \& Skoog) (Murashige and Skoog, 1962) plates with vitamins (PlantMedia) and $1 \%(\mathrm{w} / \mathrm{v})$ sucrose, unless indicated otherwise. The plates were kept at $4^{\circ} \mathrm{C}$ in darkness for 2 days, and then moved into a growth room. Rice plants was grown at $28^{\circ} \mathrm{C}$, and Arabidopsis plants at $20^{\circ} \mathrm{C}$, with a $16 \mathrm{~h} / 8 \mathrm{~h}$ (light/darkness) photoperiod.

\section{Phylogenetic Analysis}

Closely related Arabidopsis and rice Aux/IAA proteins to OsIAA9 were identified by BLAST searching Arabidopsis and 
rice proteome database ${ }^{1}$ using the entire amino acid sequence of OsIAA9. Full-length amino acid sequences of OsIAA9 and closely related Arabidopsis and rice Aux/IAA proteins were subjected to phylogenetic analysis using "One Click" mode with default settings on Phylogeny ${ }^{2}$.

\section{Auxin Treatment}

To examine the expression of OsIAA9 in response to auxin, 10day-old rice seedlings were treated with $10 \mu \mathrm{M}$ IAA for $4 \mathrm{~h}$ in darkness on a shaker at $40 \mathrm{rpm}$. Samples were frozen in liquid $\mathrm{N}_{2}$ and kept at $-80^{\circ} \mathrm{C}$ for RNA isolation.

\section{RNA Isolation and RT-PCR}

Total RNA from rice and Arabidopsis seedlings was isolated by using the procedures described previously (Wang et al., 2014; Guo et al., 2015; Liu et al., 2015). cDNA was synthesized by Oligo(dT)-primed reverse transcription using the EazyScript First-Strand DNA Synthesis Super Mix (TransGen Biotech) according to the manufacturer's instructions.

RT-PCR was used to examine the expression of OsIAA9, and Arabidopsis gene ACTIN2 (ACT2) or rice gene OsACT2 were used as controls for RT-PCR.

\section{Constructs}

The reporters constructs LexA-Gal4:GUS and Gal4:GUS, and the effector constructs GD, LD-VP,CAT, and $A R F$ s used for protoplast transfection were as described previously (Tiwari et al., 2003; Wang et al., 2005b, 2007; Liu et al., 2015).

To generate $\mathrm{HA}$ or GD tagged OsIAA9 constructs for protoplast transfection assays, the full-length open-reading frame (ORF) of OsIAA9 was amplified by RT-PCR using RNA isolated from rice seedlings, and cloned in frame with an $\mathrm{N}$-terminal HA or GD tag into the pUC19 vector under the control of the $35 \mathrm{~S}$ promoter (Tiwari et al., 2003; Wang et al., 2005b). The 35S:OsIAA9 construct in pUC19 was digested with proper enzymes, and subcloned into the binary vector $p P Z P 211$ (Hajdukiewicz et al., 1994) for plant transformation.

To generate GD tagged OsIAA9CTD for protoplast transfection, ORF sequence of OsIAA9CTD (corresponding to the amino acid residues 84-182) was amplified by PCR using 35S:OsIAA9 plasmids as template, and cloned in frame with an $\mathrm{N}$-termianl GD tag into the pUC19 vector under the control of the $35 S$ promoter.

To generate GFP tagged OsIAA9 for subcellular localization analysis of OsIAA9, GD tag in 35S:GD-OsIAA9 construct was replaced with a GFP tag, subcloned into the binary vector pPZP211, and used for plant transformation.

The primers used for gene cloning and gene expression analysis of OsIAA9 are OsIAA9-F, 5'-CAACATATGGAGCT GGAGCTTGGGCT-3', OsIAA9- $R, 5^{\prime}$ - CAACTTAAGTTAACC CAGTATCTTCAGGC - $3^{\prime}$, and OsIAA9CTD-F, 5' -CAACATAT GTCGGCGCGGCGGGCGT-3'. The primers used to amplify ACT2 and OsACT2 were described previously (Guo et al., 2015).

${ }^{1}$ http://phytozome.jgi.doe.gov/pz/portal.html

${ }^{2}$ www.phylogeny.fr

\section{Plant Transformation and Transgenic Plants Selection}

About 5-week-old Arabidopsis plants with several mature flowers on the main inflorescence were transformed using the floral dip method (Clough and Bent, 1998). T1 seeds were sterilized and grown on $1 / 2 \mathrm{MS}$ plates containing $50 \mu \mathrm{g} / \mathrm{ml}$ kanamycin to select transgenic plants. At least five transgenic lines with similar phenotypes were obtained. Phenotypes of transgenic plants were examined in the T1 generation, and confirmed in following several generations. Represent homozygous T3 or T4 transgenic plants were used for further analysis.

\section{Plasmid DNA Isolation, Protoplasts Isolation, Transfection, and GUS Activity Assays}

The procedures for plasmids preparation, protoplast isolation, transfection, and GUS activity assay have been described previously (Wang et al., 2005b, 2007, 2008, 2014, 2015; Zhou et al., 2014; Guo et al., 2015; Liu et al., 2015). Briefly, plasmids of the reporter and effector genes were prepared using the GoldHi EndoFree Plasmid Maxi Kit (Kangwei), and co-trasfected into protoplasts isolated from rosette leaves of $\sim 4$-week-old Col wild type Arabidopsis plants. The transfected protoplasts were incubated under darkness at room temperature for $20-22 \mathrm{~h}$ before GUS activities were measured using a SynergyTM HT microplate reader (BioTEK).

\section{Gravitropic Response Assays}

Gravitropic response was measured as described by Li et al. (2011). Briefly, sterilized seeds were grown vertically on $1 / 5$ MS plates for 4 days. The plates were photographed and the plates were then turned $90^{\circ}$. The plates were photographed again 1 day later. Angles of the angle between gravity and the root were measured using NIH Image $\mathrm{J}^{3}$.

\section{Starch Granule Staining}

Starch granule was stained by following the procedures described by Li et al. (2011).

\section{Microscopy}

Photographs of Arabidopsis seedlings were taken under a Motic $\mathrm{K}$ dissection microscope equipped with an EOS 1100D camera. Root length was then measured using NIH Image J. GFP florescence of OsIAA9-GFP transgenic Arabidopsis seedlings was examined under an Olympus FV1000 confocal microscope.

\section{RESULTS}

\section{OsIAA9 is an Auxin Response Gene That Encodes a Canonical Aux/IAA Protein}

Available experimental evidences suggest that functional mechanism of Aux/IAA may be conserved in rice and

${ }^{3}$ http://rsbweb.nih.gov/ij 
Arabidopsis. To test if this is the case, we decided to identify a canonical rice $A u x / I A A$ gene, and study its functions in the regulation of auxin signaling and plant growth and development in Arabidopsis.

OsIAA9 was chosen because it is one of the Aux/IAA genes whose expression was highly induced by $2,4-\mathrm{D}$, a synthesized auxin (Jain et al., 2006). As shown in Figure 1A, expression of OsIAA9 was also highly induced by IAA, a natural occurred auxin. By using the entire amino acid sequence of OsIAA9 to BLAST rice and Arabidopsis protein database 4 , we identified and selected several Aux/IAA proteins that have relative higher amino acid sequence similarity to OsIAA9. Phylogenetic analysis showed that OsIAA9 and OsIAA20 are paralogs. The most closely related Arabidopsis Aux/IAA to OsIAA9 is IAA31 (Figure 1B), an Aux/IAA

${ }^{4}$ http://phytozome.jgi.doe.gov/pz/portal.html protein with mutated Domain II (Figure 1C). These results are largely consistent with that reported by Jain et al. (2006).

Previously experiment showed that canonical Aux/IAA proteins contain an $\mathrm{LxLxL}$ repressor motif in Domain I, a GWPPV degron core sequence in Domain II, conserved KR residues between Domain I and Domain II that are crucial for the degradation of Arabidopsis Aux/IAA proteins, some conserved amino acid residues in Domain III and Domain IV that are require for protein-protein interaction among Aux/IAA proteins or between Aux/IAA proteins and ARFs (Tiwari et al., 2001, 2003, 2004; Dreher et al., 2006; Nanao et al., 2014). As shown in Figure 1C, OsIAA9 has all the features for a canonical Aux/IAA protein. In addition, OsIAA9 also has the conserved $\mathrm{W}$ residue found in OsIAAs and OsARFs that is crucial for protein-protein interaction ( $\mathrm{Ni}$ et al., 2014).

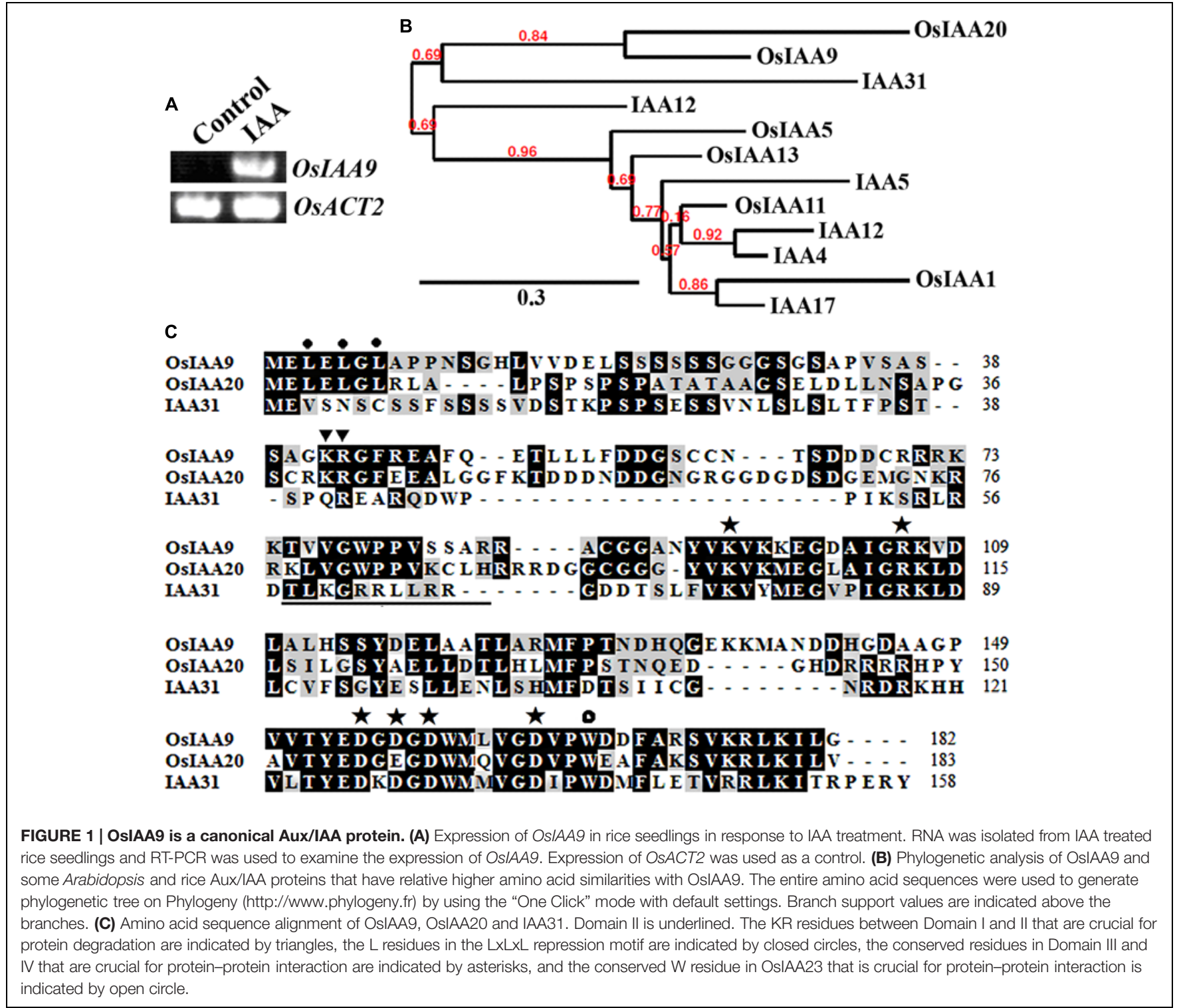




\section{OsIAA9 is a Transcription Repressor and its Stability is Affected by Auxin}

In Arabidopsis, canonical Aux/IAA proteins are short-lived proteins whose stability is affected by auxin, and they function as transcription repressors. To examine if OsIAA9 is a transcription repressor and its stability is affected by auxin, we first examine it subcellular localization by examining transgenic Arabidopsis plants expressing GFP-OsIAA9 under the control of the $35 \mathrm{~S}$ promoter. As shown in Figure 2, OsIAA9 is predominantly localized in nucleus.

We then examined if OsIAA9 functions as transcriptional repressor by using protoplast transfection assays. Plasmids of effector gene $G D$-OSIAA9 or control gene $G D$, activator gene $L D$ $V P$, and the reporter gene LexA-GAL4:GUS were contransfected into protoplasts, and GUS activities were measured after the transfected protoplasts were incubated in the presence and absent of $1.0 \mu \mathrm{M}$ IAA. As shown in Figure 3A, in the absence of IAA, co-transfection of control gene $G D$ and activator gene $L D-V P$ activated the reporter gene, while co-transfection of effector gene $G D-O S I A A 9$ and activator gene $L D-V P$ resulted in repression of

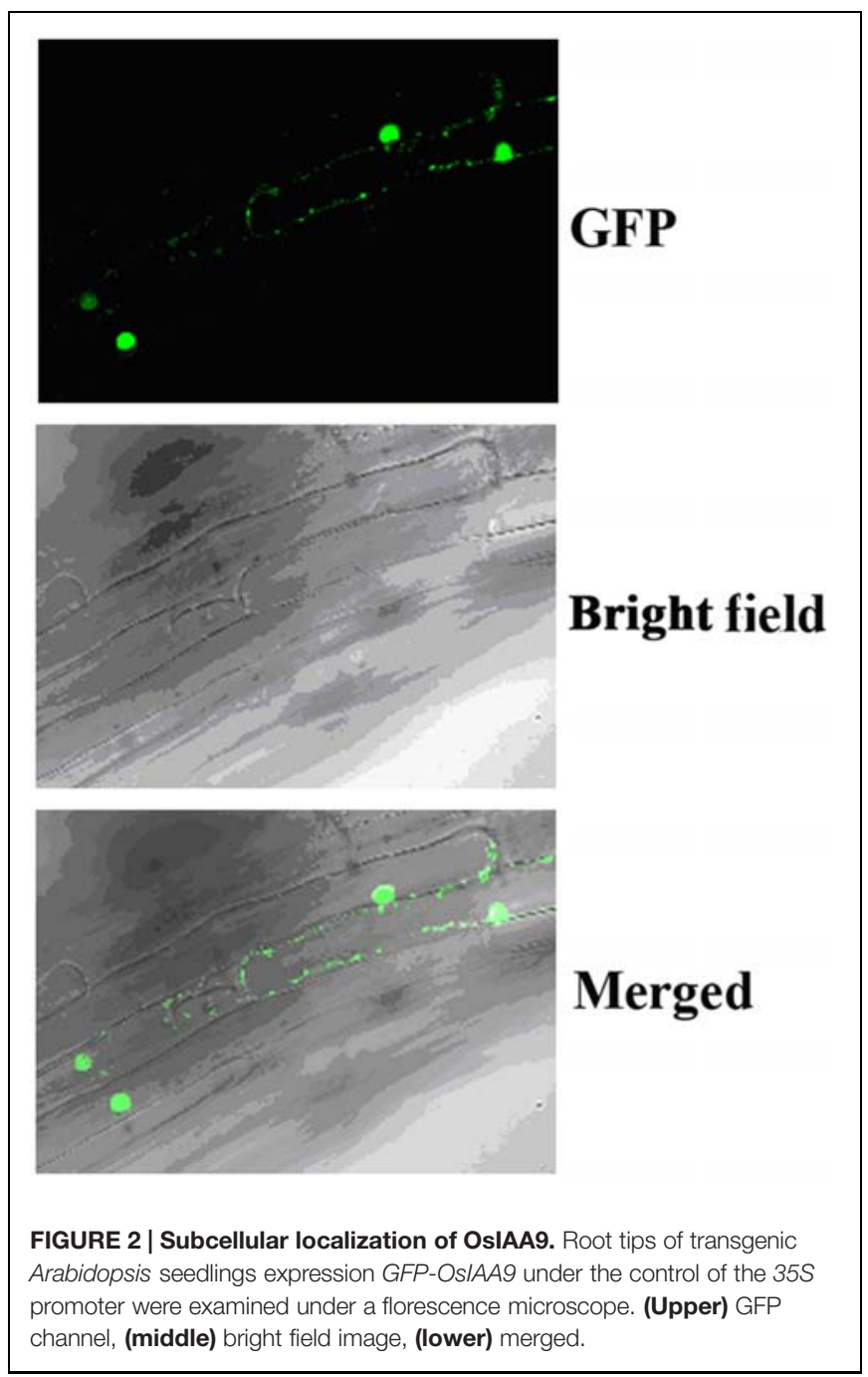

the reporter gene. In the presence of IAA, the repression on the expression of the reporter gene by co-transfected OsIAA9 gene was partially released (Figure 3A), indicating that OsIAA9 is a transcription repressor, and it is unstable in the presence of auxin. When transfected into protoplasts with an integrated auxin response reporter gene DR5:GUS, OsIAA9 repressed the expression of the reporter gene (Figure 3B), suggesting that OsIAA9 regulates auxin response gene expression.

\section{OsIAA9 Interacts With ARF5 in Plant Cells}

In Arabidopsis, Aux/IAA proteins regulate auxin signaling through interacting with ARF activators. So far only five ARF
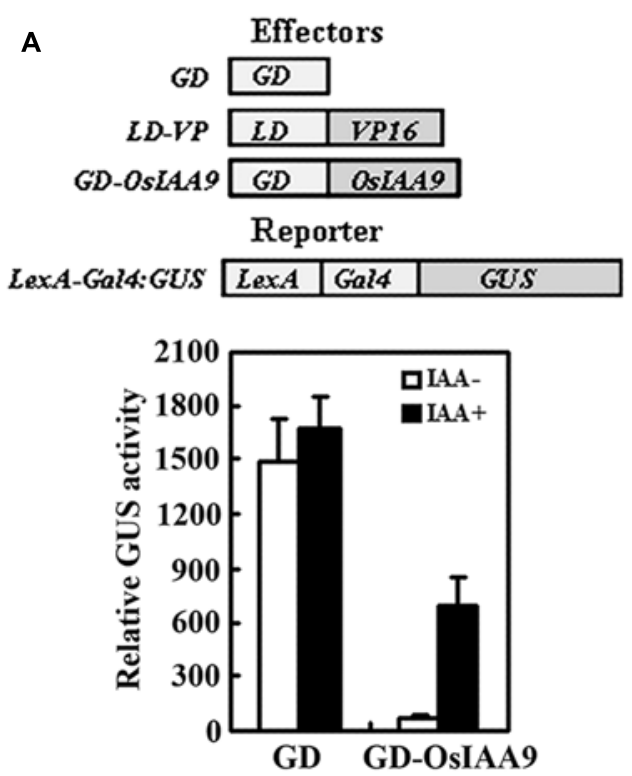

B

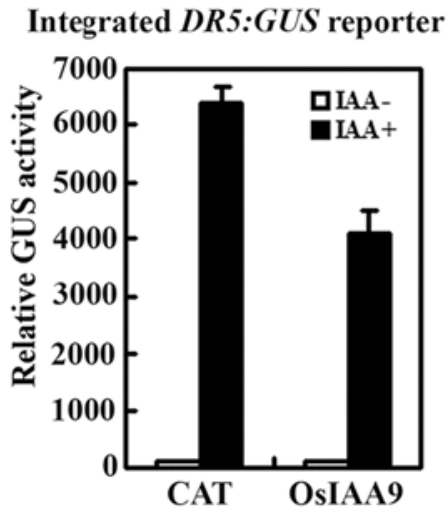

FIGURE 3 | OsIAA9 is a transcriptional repressor and its stability is affected by auxin. Repression of LexA-Ga/4:GUS reporter gene (A), and the integrated DR5:GUS reporter gene (B) by OsIAA9. Effectors and reporters (drawn on the top of $\mathbf{A}$ ) or effectors plasmids alone were transfected into protoplasts isolated from Col wild type, or DR5:GUS transgenic plants, and incubated in the presence and absence of $1 \mu \mathrm{M}$ IAA for 20-22 h, then GUS activity was assayed. The 35S:CAT (chloramphenicol acetyltransferase) plasmids were used as a control. Data represent the mean \pm SD of three replicates. 
activators in Arabidopsis have been shown to be transcriptional activators (Wang et al., 2005b). Having shown that OsIAA9 is a transcriptional repressor and it regulates auxin reporter gene expression (Figure 3), we wanted to further examine if OsIAA9 interacts with any of the ARF activators by using protoplast transfection assays.

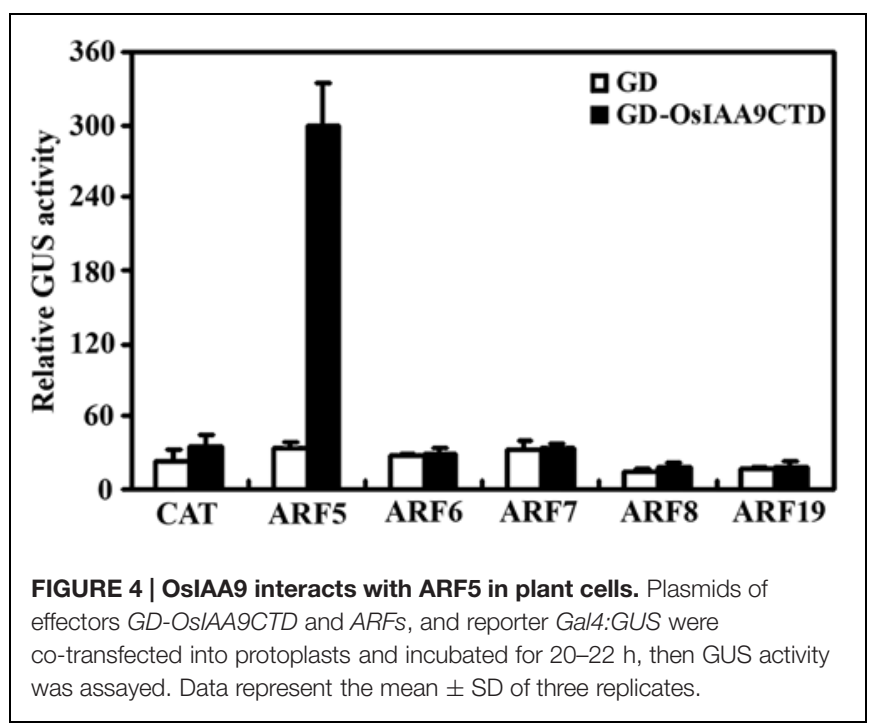

Because Aux/IAA proteins have been shown to be active transcription repressor, and Domain III and IV are the domains that required for the interaction among Aux/IAA proteins and between Aux/IAA protein and ARFs, we made a GD-OsIAA9CTD construct by fusing OsIAA9 C-terminal Domain (Domain III and IV) with GD, and cotransfected it with ARF activator effector genes and the reporter gene Gal4:GUS into protoplasts. GD gene was cotransfected as a control. GUS activity was measured after incubation. As shown in Figure 4, cotransfection of $G D$ gene with all the ARF activator genes does not have any effects on the expression of the reporter gene, while cotransfection of GD-OsIAA9CTD gene with ARF5 gene, but not other ARF activator genes activated Gal4:GUS reporter, suggesting that OsIAA9 specifically interacts with ARF5.

\section{Expression of OsIAA9 in Arabidopsis Affects Lateral Root Formation and Root Gravitropic Response}

The results described above indicate that OsIAA9 is a canonical Aux/IAA protein. Arabidopsis transgenic plants overexpressing wild type Arabidopsis and grape Aux/IAA genes are morphologically similar to wild type plants (Park et al., 2002; Fujita et al., 2012; Kohno et al., 2012). However, expression of PtrIAA14.1, a canonical Aux/IAA protein encoding wild type poplar Aux/IAA gene in Arabidopsis resulted in auxinrelated phenotypes including semi-draft with increased number

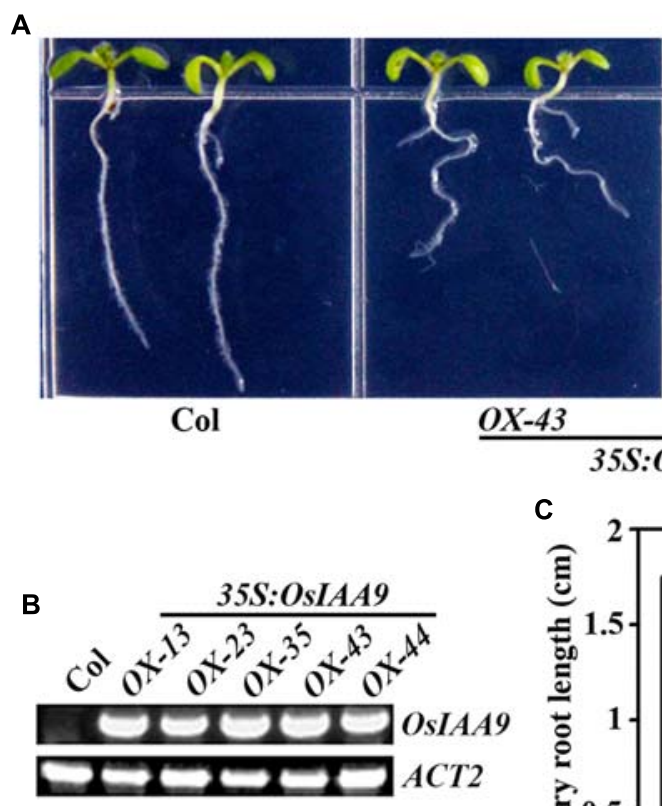

A
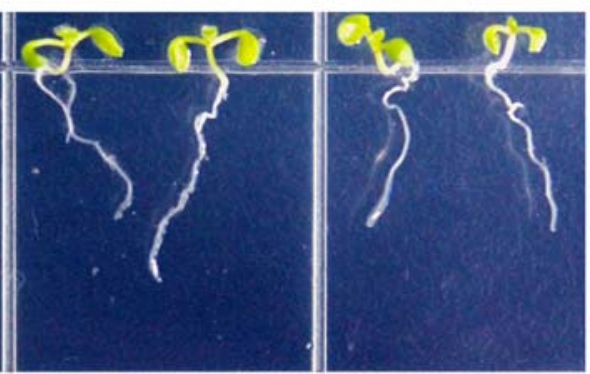

35S:OsIAA9

35S:GFP-OSIAA9
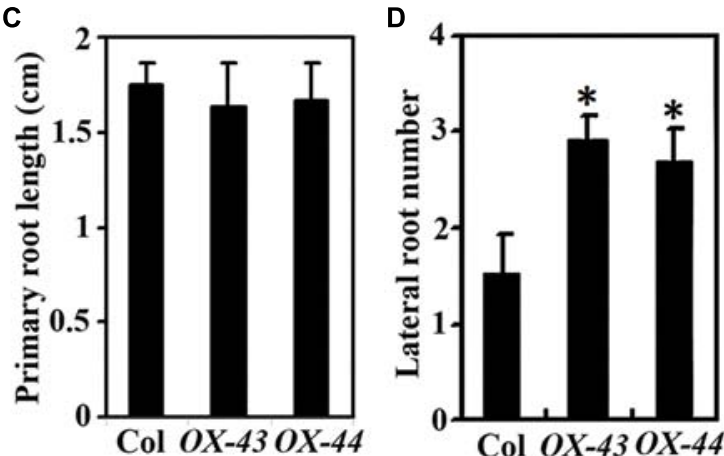

FIGURE 5 | Morphologies of Arabidopsis transgenic plant seedlings expressing OsIAA9. (A) Seven-day-old Col wild type and transgenic plant seedlings. (B) Expression of OsIAA9 in transgenic plants. RNA was isolated from OsIAA9 transgenic Arabidopsis seedlings and RT-PCR was used to examine the expression of OsIAA9. Expression of ACT2 was used as a control. (C) Primary root length of 7-day-old wild type and transgenic plants. (D) Lateral root number of 10-day-old wild type and transgenic plants. Data in (C) and (D) represent the mean \pm SD of $12-15$ seedlings. ${ }^{*}$, significantly different from Col wild type seedlings ( $\left.p<0.0001\right)$. 


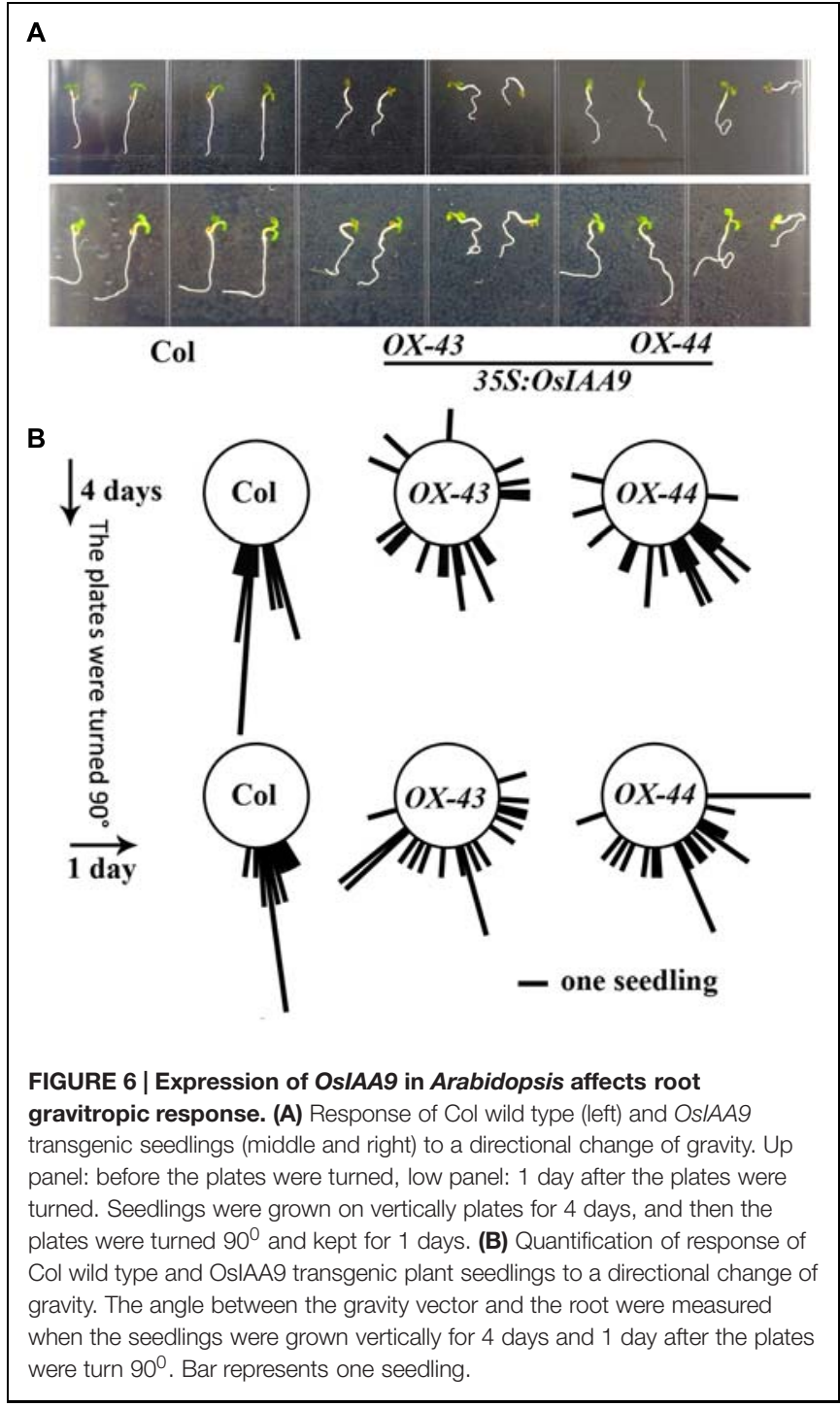

of branches, down-curling leaves, and greatly reduced fertility (Liu et al., 2015). To further explore if OsIAA9 functions similar to canonical Aux/IAA proteins in Arabidopsis, we generated transgenic plant expressing OsIAA9 under the control of the $35 S$ promoter (35S:OsIAA9). As shown in Figure 5A, transgenic Arabidopsis plants have reduced root gravitropic response as indicated by the root orientations. Expression of OsIAA9 in the transgenic plants was confirmed by RT-PCR (Figure 5B). Quantitative analysis showed that root elongation is largely unaffected in the Arabidopsis transgenic plants expressing OsIAA9 (Figure 5C). However, the transgenic plants produced more lateral roots (Figure 5D).

Transgenic plants expressing GFP-OsIAA9 also resulted in abnormal root gravitropic response (Figure 5A), indicating that GFP-OsIAA9 protein is functional, thus the plants were used to examine subcellular localization of OsIAA9 (Figure 2).

To further examine the roles of OsIAA9 in regulating root gravitropic response, we tested the gravitropic response of Col wild type and OsIAA9 transgenic Arabidopsis seedlings to

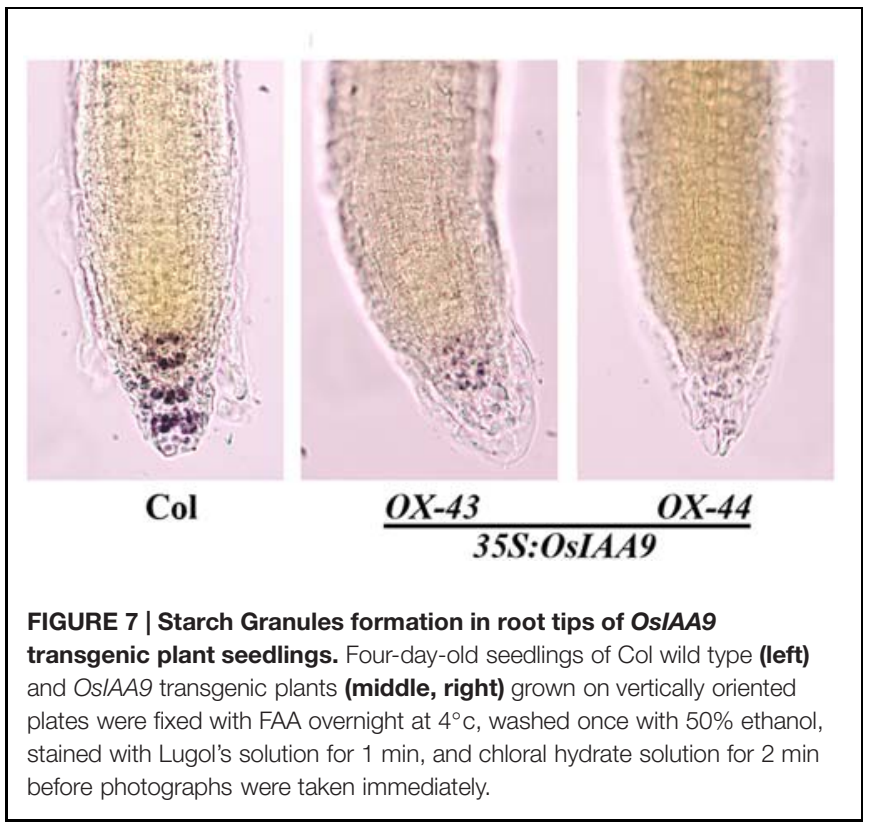

changed gravity. Seedlings were grown on vertical plates for 4 days, and pictured to measure the root directions. The plates were then turned $90^{\circ}$ to change the gravity, photographs were taken again after $24 \mathrm{~h}$. As shown in Figure 6A, wild type seedlings grew straight down toward the gravity direction before and after the plates were turned, whereas roots of transgenic seedling were in random directions before and after the plates were turned. Quantitative results of the root direction further confirmed our observation (Figure 6B).

\section{Starch Granules Accumulation is Affected in the OsIAA9 Transgenic Arabidopsis Seedlings}

Starch granules in root tips play an important role during the sensing of gravity stimulus. Because the root gravitropic response was altered in the OSIAA9 transgenic plants, we suspected that starch granule formation in the transgenic plants may be disrupted. To test if this is the case, we compared starch granule formation in wild type and the transgenic Arabidopsis seedlings by staining the roots with Lugol's solution, which contains iodine that can react with starch granules to generate a visible bright blue color. We found that in wild-type root tips, strong staining was observed in several different layers of columella cells, while in root tips of the OsIAA9 transgenic Arabidopsis seedlings, only slight staining was observed, and there is no clear stained cell layers could be observed (Figure 7).

\section{DISCUSSION}

Interplay of Aux/IAA proteins and ARFs regulates auxin signaling in Arabidopsis (Hagen and Guilfoyle, 2002). Characterization of gain-of-function mutants in Arabidopsis revolved that $A u x / I A A$ genes regulated many aspects of plant 
growth and development (Nagpal et al., 2000; Rogg et al., 2001; Fukaki et al., 2002; Uehara et al., 2008; Ploense et al., 2009; Rinaldi et al., 2012). So far all experimental evidences suggest that Aux/IAA proteins in Arabidopsis and rice may regulate auxin signaling and plant growth and development in a similar manner (Jain et al., 2006; Nakamura et al., 2006; Jun et al., 2011; Zhu et al., 2011; Kitomi et al., 2012; Song and Xu, 2013). Our results in this report show that OsIAA9 is a canonical Aux/IAA protein, it regulates auxin signaling in a way similar to that of the canonical Aux/IAA proteins in Arabidopsis. However, unlike the Arabidopsis canonical Aux/IAA proteins have been studied so far, OsIAA9 regulates lateral root formation and root gravitropic response when expressed in Arabidopsis in an unmutated form.

\section{OsIAA9 is a Canonical Aux/IAA Protein}

All canonical Aux/IAA proteins contain four conserved domains. An LxLxL motif-containing domain, a degron-containing domain, and two domains required for protein-protein interaction (Ulmasov et al., 1997, 1999; Ramos et al., 2001; Tiwari et al., 2001, 2003, 2004; Dreher et al., 2006; Jain et al., 2006; Nanao et al., 2014).

Bioinformatics analysis showed that OsIAA9 is closely related to IAA31, a Domain II mutated type Arabidopsis Aux/IAA protein (Figure 1B). However, it contains all the features of a canonical Aux/IAA protein has, including the conserved KR residues crucial for 26 proteasome degradation of Aux/IAA proteins (Dreher et al., 2006), and the conserved W residue crucial for protein-protein interactions of OsIAAs and OsARFs (Ni et al., 2014) (Figure 1B). In consist with the canonical Aux/IAA protein features it has, OsIAA9 functions as a transcription repressor, its stability is affected by Auxin, it regulates auxin response repoter gene expression (Figure 3), and it interacted with ARF5 in transfected protoplasts (Figure 4). These results suggest that OsIAA9 is a canonical Aux/IAA protein, it regulates auxin signaling in a way similar to all other canonical Aux/IAA proteins.

\section{Expression of Unmutated OsIAA9 in Arabidopsis Resulted in Auxin Related Phenotypes}

So far all the aux/iaa mutants identified in Arabidopsis and rice with phenotypes are gain-of-function mutants with mutations occurred within Domain II of corresponding Aux/IAA proteins (Tian and Reed, 1999; Nagpal et al., 2000; Rogg et al., 2001; Fukaki et al., 2002; Tian et al., 2002; Knox et al., 2003; Uehara et al., 2008; Ploense et al., 2009; Jun et al., 2011; Zhu et al., 2011; Kitomi et al., 2012; Rinaldi et al., 2012). On the other hand, expression

\section{REFERENCES}

Bassa, C., Mila, I., Bouzayen, M., and Audran-Delalande, C. (2012). Phenotypes associated with down-regulation of Sl-IAA27 support functional diversity among Aux/IAA family members in tomato. Plant Cell Physiol. 53, 1583-1595. doi: $10.1093 / \mathrm{pcp} / \mathrm{pcs} 101$

Clough, S. J., and Bent, A. F. (1998). Floral dip: a simplified method for Agrobacterium-mediated transformation of Arabidopsis thaliana. Plant J. 16, 735-743. doi: 10.1046/j.1365-313x.1998.00343.x of mutated type, but not wild type Arabidopsis Aux/IAA genes in Arabidopsis resulted in auxin-related phenotypes (Park et al., 2002; Fukaki et al., 2005; Sato and Yamamoto, 2008). Expression of mutated or dominant mutation-type rice $A u x / I A A$ genes in rice also resulted in auxin-related phenotypes (Nakamura et al., 2006; Song and Xu, 2013), suggest that stability of Aux/IAA proteins are crucial for their functions in regulating plant growth and development, and that function mechanisms of Arabidopsis and rice Aux/IAA proteins may be conserved.

Similar to other canonical Aux/IAA proteins, OsIAA9 functions as a transcription repressor, and its stability is affected by auxin as mentioned above. However, transgenic Arabidopsis plants expressing OsIAA9 showed several auxinrelated phenotypes, including increased lateral root formation and reduced root gravitropic response (Figures 5 and 6). Previously we have showed that expression of wild type PtrIAA14.1, a canonical Aux/IAA gene from poplar, resulted in phenotypes changes in Arabidopsis (Liu et al., 2015), but the phenotypes are different from that of Arabidopsis transgenic plants expressing OsIAA9. On the other hand, among all the five ARF activators, both PtrIAA14.1 and OsIAA9 interacted only with ARF5 in transfected protoplasts (Liu et al., 2015; Figure 4), indicating that interaction between ARF5 and OsIAA9 may contribute little, if any to the resulted phenotypes in the transgenic plants.

In summary, we showed that OsIAA9 is a canonical Aux/IAA protein, and it causes auxin-related phenotypes including lateral root formation and gravitropic response when expressed in Arabidopsis.

\section{AUTHOR CONTRIBUTIONS}

SW conceived the study. SL and SW designed the experiments. SL, QL, SL, NMP, and HT performed the experiments. SL and SW analyzed the data. SW drafted the manuscript. All authors read and approved the final manuscript.

\section{ACKNOWLEDGMENTS}

This work was supported by the Key Laboratory of Molecular Epigenetics of MOE (130014542), the Department of Human Resources and Social Security of Jilin Province (http://hrss.ji.gov.cn) and the Programme for Introducing Talents to Universities (B07017). The funders had no role in study design, data collection and analysis, decision to publish, or preparation of the manuscript.

Deng, W., Yang, Y., Ren, Z., Audran-Delalande, C., Mila, I., Wang, X., et al. (2012). The tomato SIIAA15 is involved in trichome formation and axillary shoot development. New Phytol. 194, 379-390. doi: 10.1111/j.1469-8137.2012. 04053.x

Dharmasiri, N., Dharmasiri, S., and Estelle, M. (2005). The F-box protein TIR1 is an auxin receptor. Nature 435, 441-445. doi: 10.1038/nature03543

Dreher, K. A., Brown, J., Saw, R. E., and Callis, J. (2006). The Arabidopsis Aux/IAA protein family has diversified in degradation and auxin responsiveness. Plant Cell 18, 699-714. doi: 10.1105/tpc.105.039172 
Fukaki, H., Nakao, Y., Okushima, Y., Theologis, A., and Tasaka, M. (2005). Tissue-specific expression of stabilized SOLITARY-ROOT/IAA14 alters lateral root development in Arabidopsis. Plant J. 44, 382-395. doi: 10.1111/j.1365313X.2005.02537.x

Fukaki, H., Tameda, S., Masuda, H., and Tasaka, M. (2002). Lateral root formation is blocked by a gain-of-function mutation in the SOLITARY-ROOT/IAA14 gene of Arabidopsis. Plant J. 29, 153-168. doi: 10.1046/j.0960-7412.2001.01201.x

Fujita, K., Horiuchi, H., Takato, H., Kohno, M., and Suzuki, S. (2012). Auxinresponsive grape Aux/IAA9 regulates transgenic Arabidopsis plant growth. Mol. Biol. Rep. 39, 7823-7829. doi: 10.1007/s11033-012-1625-9

Guilfoyle, T. J. (2015). The PB1 domain in auxin response factor and Aux/IAA proteins: a versatile protein interaction module in the auxin response. Plant Cell 27, 33-43. doi: 10.1105/tpc.114.132753

Guilfoyle, T. J., and Hagen, G. (2007). Auxin response factors. Curr. Opin. Plant Biol. 10, 453-460. doi: 10.1016/j.pbi.2007.08.014

Guo, H., Zhang, W., Tian, H., Zheng, K., Dai, X., Liu, S., et al. (2015). An auxin responsive CLE gene regulates shoot apical meristem development in Arabidopsis. Front. Plant Sci. 6:295. doi: 10.3389/fpls.2015.00295

Hagen, G., and Guilfoyle, T. J. (2002). Auxin-responsive gene expression: genes, promoters, and regulatory factors. Plant Mol. Biol. 49, 373-385. doi: 10.1023/A:1015207114117

Hajdukiewicz, P., Svab, Z., and Maliga, P. (1994). The small, versatile pPZP family of Agrobacterium binary vectors for plant transformation. Plant Mol. Biol. 25, 989-994. doi: 10.1007/BF00014672

Jain, M., Kaur, N., Garg, R., Thakur, J. K., Tyagi, A. K., and Khurana, J. P. (2006). Structure and expression analysis of early auxin-responsive Aux/IAA gene family in rice (Oryza sativa). Funct. Integr. Genomics 6, 47-59. doi: $10.1007 /$ s10142-005-0142-5

Jun, N., Gaohang, W., Zhenxing, Z., Huanhuan, Z., Yunrong, W., and Ping, W. (2011). OsIAA23-mediated auxin signaling defines postembryonic maintenance of QC in rice. Plant J. 68, 433-442. doi: 10.1111/j.1365313X.2011.04698.X

Kepinski, S., and Leyser, O. (2005). The Arabidopsis F-box protein TIR1 is an auxin receptor. Nature 435, 446-451. doi: 10.1038/nature03542

Kitomi, Y., Inahashi, H., Takehisa, H., Sato, Y., and Inukai, Y. (2012). OsIAA13mediated auxin signaling is involved in lateral root initiation in rice. Plant Sci. 190, 116-122. doi: 10.1016/j.plantsci.2012.04.005

Knox, K., Grierson, C. S., and Leyser, O. (2003). AXR3 and SHY2 interact to regulate root hair development. Development 130, 5769-5777. doi: 10.1242/dev.00659

Kohno, M., Takato, H., Horiuchi, H., Fujita, K., and Suzuki, S. (2012). Auxinnonresponsive grape Aux/IAA19 is a positive regulator of plant growth. Mol Biol. Rep. 39, 911-917. doi: 10.1007/s11033-011-0816-0

Li, Y., Dai, X., Cheng, Y., and Zhao, Y. (2011). NPY genes play an essential role in root gravitropic responses in Arabidopsis. Mol. Plant 4, 171-179. doi: $10.1093 / \mathrm{mp} / \mathrm{ssq} 052$

Liu, S., Hu, Q., Luo, S., Li, Q., Yang, X., Wang, X., et al. (2015). Expression of wild-type PtrIAA14.1, a poplar Aux/IAA gene causes morphological changes in Arabidopsis. Front. Plant Sci. 6:388. doi: 10.3389/fpls.2015.00388

Murashige, T., and Skoog, F. (1962). A revised medium for rapid growth and bio-assays with tobacco tissue cultures. Physiol. Plant. 15, 473-497. doi: 10.1111/j.1399-3054.1962.tb08052.x

Nagpal, P., Walker, L. M., Young, J. C., Sonawala, A., Timpte, C., Estelle, M., et al. (2000). AXR2 encodes a member of the Aux/IAA protein family. Plant Physiol. 123, 563-574. doi: 10.1104/pp.123.2.563

Nakamura, A., Umemura, I., Gomi, K., Hasegawa, Y., Kitano, H., Sazuka, T., et al. (2006). Production and characterization of auxin-insensitive rice by overexpression of a mutagenized rice IAA protein. Plant J. 46, 297-306. doi: 10.1111/j.1365-313X.2006.02693.x

Nanao, M. H., Vinos-Poyo, T., Brunoud, G., Thévenon, E., Mazzoleni, M., Mast, D., et al. (2014). Structural basis for oligomerization of auxin transcriptional regulators. Nat. Commun. 5:3617. doi: 10.1038/ncomms4617

Ni, J., Zhu, Z., Wang, G., Shen, Y., Zhang, Y., and Wu, P. (2014). Intragenic suppressor of osiaa23 revealed a conserved tryptophan residue crucial for protein-protein interactions. PLOS ONE 9:85358. doi: 10.1371/journal.pone. 0085358

Overvoorde, P. J., Okushima, Y., Alonso, J. M., Chan, A., Chang, C., Ecker, J. R., et al. (2005). Functional genomic analysis of the AUXIN/INDOLE-3-ACETIC
ACID gene family members in Arabidopsis thaliana. Plant Cell 17, 3282-3300. doi: $10.1105 /$ tpc. 105.036723

Park, J. Y., Kim, H. J., and Kim, J. (2002). Mutation in domain II of IAA1 confers diverse auxin-related phenotypes and represses auxin-activated expression of Aux/IAA genes in steroid regulator-inducible system. Plant J. 32, 669-683. doi: 10.1046/j.1365-313X.2002.01459.x

Ploense, S. E., Wu, M. F., Nagpal, P., and Reed, J. W. (2009). A gain-offunction mutation in IAA18 alters Arabidopsis embryonic apical patterning. Development 136, 1509-1517. doi: 10.1242/dev.025932

Ramos, J. A., Zenser, N., Leyser, O., and Callis, J. (2001). Rapid degradation of auxin/indoleacetic acid proteins requires conserved amino acids of domain II and is proteasome dependent. Plant Cell 13, 2349-2360. doi: 10.2307/ 3871512

Rinaldi, M. A., Liu, J., Enders, T. A., Bartel, B., and Strader, L. C. (2012). A gainof-function mutation in IAA16 confers reduced responses to auxin and abscisic acid and impedes plant growth and fertility. Plant Mol. Biol. 79, 359-373. doi: 10.1007/s11103-012-9917-y

Rogg, L. E., Lasswell, J., and Bartel, B. (2001). A gain-of-function mutation in IAA28 suppresses lateral root development. Plant Cell 13, 465-480. doi: 10.1105/tpc.13.3.465

Sato, A., and Yamamoto, K. T. (2008). Overexpression of the non-canonical Aux/IAA genes causes auxin-related aberrant phenotypes in Arabidopsis. Physiol. Plant. 133, 397-405. doi: 10.1111/j.1399-3054.2008.01055.x

Song, Y., and Xu, Z. F. (2013). Ectopic overexpression of an AUXIN/INDOLE3-ACETIC ACID (Aux/IAA) Gene OsIAA4 in rice induces morphological changes and reduces responsiveness to auxin. Int. J. Mol. Sci. 14, 13645-13656. doi: 10.3390/ijms140713645

Su, L., Bassa, C., Audran, C., Mila, I., Cheniclet, C., Chevalier, C., et al. (2014). The auxin S1-IAA17 transcriptional repressor controls fruit size via the regulation of endoreduplication-related cell expansion. Plant Cell Physiol. 55, 1969-1976. doi: $10.1093 / \mathrm{pcp} / \mathrm{pcu} 124$

Tan, X., Calderon-Villalobos, L. I., Sharon, M., Zheng, C., Robinson, C. V., Estelle, M., et al. (2007). Mechanism of auxin perception by the TIR1 ubiquitin ligase. Nature 446, 640-645. doi: 10.1038/nature05731

Tian, Q., and Reed, J. W. (1999). Control of auxin-regulated root development by the Arabidopsis thaliana SHY2/IAA3 gene. Development 126, 711-721.

Tian, Q., Uhlir, N. J., and Reed, J. W. (2002). Arabidopsis SHY2/IAA3 inhibits auxin-regulated gene expression. Plant Cell 14, 301-319. doi: 10.1105/tpc. 010283

Tiwari, S. B., Hagen, G., and Guilfoyle, T. J. (2003). The roles of auxin response factor domains in auxin-responsive transcription. Plant Cell 15, 533-543. doi: 10.1105/tpc.008417

Tiwari, S. B., Hagen, G., and Guilfoyle, T. J. (2004). Aux/IAA proteins contain a potent transcriptional repression domain. Plant Cell 16, 533-543. doi: 10.1105/tpc.017384

Tiwari, S. B., Wang, X.-J., Hagen, G., and Guilfoyle, T. J. (2001). Aux/IAA proteins are active repressors and their stability and activity are modulated by auxin. Plant Cell 13, 2809-2822. doi: 10.1105/tpc.13.12.2809

Uehara, T., Okushima, Y., Mimura, T., Tasaka, M., and Fukaki, H. (2008). Domain II mutations in CRANE/IAA18 suppress lateral root formation and affect shoot development in Arabidopsis thaliana. Plant Cell Physiol. 49, 1025-1038. doi: $10.1093 / \mathrm{pcp} / \mathrm{pcn} 079$

Ulmasov, T., Hagen, G., and Guilfoyle, T. J. (1997). ARF1, a transcription factor that binds auxin response elements. Science 276, 1865-1868. doi: 10.1126/science. 276.5320 .1865

Ulmasov, T., Hagen, G., and Guilfoyle, T. J. (1999). Activation and repression of transcription by auxin response factors. Proc. Natl. Acad. Sci. U.S.A. 96, 5844-5849. doi: 10.1073/pnas.96.10.5844

Wang, H., Jones, B., Li, Z., Frasse, P., Delalande, C., Regad, F., et al. (2005a). The tomato Aux/IAA transcription factor IAA9 is involved in fruit development and leaf morphogenesis. Plant Cell 17, 2676-2692. doi: 10.1105/tpc.105.033415

Wang, S., Tiwari, S. B., Hagen, G., and Guilfoyle, T. J. (2005b). AUXIN RESPONSE FACTOR7 restores the expression of auxin-responsive genes in mutant Arabidopsis leaf mesophyll protoplasts. Plant Cell 17, 1979-1993. doi: 10.1105/tpc.105.031096

Wang, S., Chang, Y., Guo, J., and Chen, J. G. (2007). Arabidopsis ovate family protein 1 is a transcriptional repressor that suppresses cell elongation. Plant J. 50, 858-872. doi: 10.1111/j.1365-313X.2007.03096.x 
Wang, S., Hubbard, L., Chang, Y., Guo, J., Schiefelbein, J., and Chen, J. G. (2008). Comprehensive analysis of single-repeat R3 MYB proteins in epidermal cell patterning and their transcriptional regulation in Arabidopsis. BMC Plant Biol. 8:81. doi: 10.1186/1471-2229-8-81

Wang, S., Li, E., Porth, I., Chen, J. G., Mansfield, S. D., and Douglas, C. J. (2014). Regulation of secondary cell wall biosynthesis by poplar R2R3 MYB transcription factor PtrMYB152 in Arabidopsis. Sci. Rep. 4:5054. doi: 10.1038/srep05054

Wang, X., Wang, X., Hu, Q., Dai, X., Tian, H., Zheng, K., et al. (2015). Characterization of an activation-tagged mutant uncovers a role of GLABRA2 in anthocyanin biosynthesis in Arabidopsis. Plant J. 83, 300-311. doi: 10.1111/tpj.12887

Zhu, Z. X., Liu, Y., Liu, S. J., Mao, C. Z., Wu, Y. R., and Wu, P. (2011). A gainof-function mutation in OsIAA11 affects lateral root development in rice. Mol. Plant 5, 154-161. doi: 10.1093/mp/ssr074
Zhou, L., Zheng, K., Wang, X., Tian, H., Wang, X., and Wang, S. (2014). Control of trichome formation in Arabidopsis by poplar single-repeat R3 MYB transcription factors. Front. Plant Sci. 5:262. doi: 10.3389/fpls.2014. 00262

Conflict of Interest Statement: The authors declare that the research was conducted in the absence of any commercial or financial relationships that could be construed as a potential conflict of interest.

Copyright (c) 2015 Luo, Li, Liu, Pinas, Tian and Wang. This is an open-access article distributed under the terms of the Creative Commons Attribution License (CC BY).

The use, distribution or reproduction in other forums is permitted, provided the original author(s) or licensor are credited and that the original publication in this journal is cited, in accordance with accepted academic practice. No use, distribution or reproduction is permitted which does not comply with these terms. 